TRANSACTIONS OF THE

AMERICAN MATHEMATICAL SOCIETY

Volume 349, Number 3, March 1997, Pages 1169-1189

$\mathrm{S} 0002-9947(97) 01896-5$

\title{
MEAN-BOUNDEDNESS AND LITTLEWOOD-PALEY FOR SEPARATION-PRESERVING OPERATORS
}

\author{
EARL BERKSON AND T. A. GILLESPIE
}

\begin{abstract}
Suppose that $(\Omega, \mathcal{M}, \mu)$ is a $\sigma$-finite measure space, $1<p<\infty$, and $T: L^{p}(\mu) \rightarrow L^{p}(\mu)$ is a bounded, invertible, separation-preserving linear operator such that the linear modulus of $T$ is mean-bounded. We show that $T$ has a spectral representation formally resembling that for a unitary operator, but involving a family of projections in $L^{p}(\mu)$ which has weaker properties than those associated with a countably additive Borel spectral measure. This spectral decomposition for $T$ is shown to produce a strongly countably spectral measure on the "dyadic sigma-algebra" of $\mathbb{T}$, and to furnish $L^{p}(\mu)$ with abstract analogues of the classical Littlewood-Paley and Vector-Valued M. Riesz Theorems for $\ell^{p}(\mathbb{Z})$.
\end{abstract}

\section{Introduction}

The aim of this article is to bring together the dominated ergodic theorem of [21] and the Banach space spectral theory initiated in [4], [5], [6] in order to transfer the classical Littlewood-Paley and Vector-Valued M. Riesz Theorems ([12, Theorem 7.2 .1 and $\S 6.5])$ from $\ell^{p}(\mathbb{Z})$ to $L^{p}(\mu)$, where $\mu$ is an arbitrary $\sigma$-finite measure and $1<p<\infty$. These results are obtained in $\$ \S 5,6$, after a detailed discussion of the relevant definitions and background developments in $\S \S 2,3,4$. Throughout $\S \S 3-6$ the boundedness of the discrete Hilbert transform in the presence of the $A_{p}$ condition for bilateral weight sequences ([15, Theorem 10]) plays a pivotal rôle.

Leaving precise definitions for the subsequent sections, we sketch here the shape of our main results. Suppose that $(\Omega, \mathcal{M}, \mu)$ is an arbitrary $\sigma$-finite measure space, $1<p<\infty$, and $T$ is a bounded, invertible, linear mapping of $L^{p}(\mu)$ onto $L^{p}(\mu)$ such that $T$ preserves disjointness of supports, and the linear modulus of $T$ is mean-bounded (in the sense of Theorem (3.2)-(ii) below). We show in Theorem (4.2) that $T$ is trigonometrically well-bounded-that is, $T$ can be expressed as a Riemann-Stieltjes integral in the strong operator topology having the following form

$$
T=\int_{0^{-}}^{2 \pi} e^{i t} d E(t),
$$

where $E(\cdot)$ is a unique projection-valued function of a real variable (possessing various additional properties weaker than those associated with a countably additive Borel spectral measure). This projection-valued function $E(\cdot)$ is used to obtain a

Received by the editors August 17, 1995.

1991 Mathematics Subject Classification. Primary 42A45, 42B25, 46E30, 47B40.

The work of the first author was supported by a grant from the National Science Foundation (U.S.A.). 
countably additive spectral measure on the dyadic sigma-algebra of subsets of $\mathbb{T}$ (Theorem (5.10)) from which one can infer (see Corollary (5.11) and Remark (5.12)) an analogue for $L^{p}(\mu)$ of the classical Littlewood-Paley Theorem. In Theorem (6.7) (see also Remark (6.15)), $E(\cdot)$ furnishes $L^{p}(\mu)$ with an abstract counterpart of the Vector-Valued M. Riesz Theorem.

We close this introductory section with a few observations about the relationship of our results to the transference methods which originated in [9], [10]. Such methods transfer convolution operators, and more generally multiplier operators, along with their bounds, in the context of a strongly continuous, uniformly bounded representation of a locally compact abelian group $G$ in a Lebesgue space of a measure. In the case of the space $L^{p}(\mu)(1<p<\infty)$ described above, and a single bounded, invertible linear operator $V$ mapping $L^{p}(\mu)$ onto $L^{p}(\mu)$ this context is achieved by the representation $n \in \mathbb{Z} \rightarrow V^{n}$, if and only if $V$ satisfies the powerboundedness condition $\sup _{n \in \mathbb{Z}}\left\|V^{n}\right\|<\infty$. Under the sole assumption that $V$ is power-bounded on $L^{p}(\mu)$, transference methods can be applied to show that $V$ is necessarily trigonometrically well-bounded ([6, Theorem (4.8)]), and to set up a lively interplay between $V$ and Fourier analysis (see, for instance, [6, Theorem (4.14)] and [8, p. 820]). In particular, the main results of the present article (Theorem (5.10), Corollary (5.11), and Theorem (6.7)) can be deduced for the power-bounded operator $V$ on $L^{p}(\mu)$ ([3, Theorem (2.12), Corollary (2.14), Theorem $(3.15)])$. However, as illustrated in $\S 4$ below, various examples arise naturally wherein an operator $T: L^{p}(\mu) \rightarrow L^{p}(\mu)$ of the sort described above is not power-bounded, and consequently the results of the present article can be viewed as an extension of transference theory in the direction of appropriate representations which need not be uniformly bounded. Our approach is made possible by the discovery in $[21, \S 3]$ (quoted in Theorem (3.2) below) that $T$ induces the formation of $A_{p}$ weight sequences which permit averaging techniques reminiscent of the usual transference methods to be carried out pointwise $\mu$-a.e. on $\Omega$.

\section{Preliminary Considerations With SEPARATION-PRESERVING Operators}

For ease of reference, we use this section to collect some standard facts regarding separation-preserving operators. Except as otherwise noted, all of this material can essentially be found in [16].

Definition. Suppose that $(\Omega, \mathcal{M}, \mu)$ is an arbitrary measure space, and $1 \leq p<$ $\infty$. A linear mapping $T: L^{p}(\mu) \rightarrow L^{p}(\mu)$ is said to be separation-preserving (respectively, positivity-preserving) provided that whenever $f \in L^{p}(\mu), g \in L^{p}(\mu)$, and $f g=0 \mu$-a.e. on $\Omega$ (respectively, $f \in L^{p}(\mu)$ and $f \geq 0 \mu$-a.e. on $\Omega$ ), the pointwise product $(T f)(T g)$ vanishes $\mu$-a.e. on $\Omega$ (respectively, $T f \geq 0 \mu$-a.e. on $\Omega)$.

The study of separation-preserving operators goes back as far as Banach [2, Chapter XI, §5]. One means for producing separation-preserving operators is the fact that if $T$ is an injective, positivity-preserving linear map of $L^{p}(\mu)$ onto $L^{p}(\mu)$ such that $T^{-1}$ is positivity-preserving, then $T$ must be separation-preserving (see [16, Proposition 3.1]). Another well-known source of separation-preserving operators is the fact that if $p \neq 2$, then any norm-preserving linear mapping $W$ of $L^{p}(\mu)$ into itself is separation-preserving ([18, Corollary 2.1]). In general, we have the 
following characterization of the separation-preserving property (see [16, Theorem $3.1])$.

(2.1) Scholium. Suppose that $(\Omega, \mathcal{M}, \mu)$ is an arbitrary measure space, and $1 \leq$ $p<\infty$. A bounded linear mapping $T$ of $L^{p}(\mu)$ into $L^{p}(\mu)$ is separation-preserving if and only if there is a bounded positivity-preserving linear mapping $|T|$ of $L^{p}(\mu)$ into $L^{p}(\mu)$ satisfying the following condition:

$$
\text { for every } f \in L^{p}(\mu), \quad|T f|=|T|(|f|) \text {, } \mu \text {-a.e. on } \Omega \text {. }
$$

If this is the case, then (2.2) uniquely characterizes $|T|$ among the bounded linear mappings of $L^{p}(\mu)$ into $L^{p}(\mu)$ (and $|T|$ is called the linear modulus of $T$ ). Moreover, $|T|$ has the property that

$$
\text { for every } f \in L^{p}(\mu), \quad|T f|=|| T|(f)|, \mu \text {-a.e. on } \Omega \text {. }
$$

(Hence, in particular, $\||T|(f)\|_{p}=\|T f\|_{p}$.)

Notice that if $T$ is both separation-preserving and positivity-preserving, then by (2.2), $T=|T|$. The well-known general relationships between invertibility and the separation-preserving property can be summarized as follows.

(2.3) Scholium. Suppose that $(\Omega, \mathcal{M}, \mu)$ is an arbitrary measure space, $1 \leq p<$ $\infty$, and $T$ is a bounded invertible separation-preserving linear mapping of $L^{p}(\mu)$ onto $L^{p}(\mu)$. Then $T^{-1}$ is separation-preserving, $|T|$ is an invertible linear mapping of $L^{p}(\mu)$ onto $L^{p}(\mu)$, and

$$
|T|^{-1}=\left|T^{-1}\right|
$$

Proof. Suppose $f \in L^{p}(\mu), g \in L^{p}(\mu)$, and $f g=0 \mu$-a.e. Put $F=T^{-1} f, G=$ $T^{-1} g, h=\min \{|F|,|G|\}$. Since $0 \leq h \leq|F|,|G|$, and since $|T|$ is positivitypreserving, we have

$$
0 \leq|T|(h) \leq \min \{|T|(|F|),|T|(|G|)\} .
$$

But

$$
\begin{aligned}
& |T|(|F|)=|T(F)|=\left|T T^{-1} f\right|=|f| ; \\
& |T|(|G|)=|g| .
\end{aligned}
$$

Hence we infer with the aid of (2.4) that

$$
0=|T|(h)=|T(h)|, \mu \text {-a.e. }
$$

Since $T$ is injective, this shows that $h=0 \mu$-a.e. In other words, $F G=0 \mu$-a.e., and hence $T^{-1}$ is separation-preserving

If $f \in L^{p}(\mu)$, and $f \geq 0$, then

$$
f=\left|T^{-1} T f\right|=\left|T T^{-1} f\right|=\left|T^{-1}\right|(|T| f)=|T|\left(\left|T^{-1}\right| f\right) .
$$

Hence $\left|T^{-1}\right||T|=|T|\left|T^{-1}\right|=I$.

Henceforth we shall assume that the measure space $(\Omega, \mathcal{M}, \mu)$ is $\sigma$-finite, and we shall employ the following terminology and notation from [16].

(2.5) Definition. A $\sigma$-endomorphism of the measure algebra $(\Omega, \mathcal{M}, \mu)$ is a mapping $\Phi: \mathcal{M} \rightarrow \mathcal{M}$ (modulo $\mu$-null sets) such that:

(i) $\Phi\left(\bigcup_{n=1}^{\infty} E_{n}\right)=\bigcup_{n=1}^{\infty} \Phi E_{n}$, for every disjoint sequence $\left\{E_{n}\right\}_{n=1}^{\infty} \subseteq \mathcal{M}$;

(ii) $\Phi(\Omega \backslash E)=\Phi(\Omega) \backslash \Phi(E)$, for all $E \in \mathcal{M}$; 
(iii) whenever $E \in \mathcal{M}$ and $\mu(E)=0$, then $\mu(\Phi E)=0$.

We shall symbolize the characteristic function of a subset $A$ of $\Omega$ by $\chi_{A}$. As described in $[16, \S 4]$, adaptation of the reasoning in $[18$, p. 461] and $[11$, pp. 453454 ] shows that a $\sigma$-endomomorphism $\Phi$ induces a unique linear operator (also denoted by $\Phi)$ from the space of all complex-valued $\mu$-measurable functions into itself such that $\Phi\left(\chi_{E}\right)=\chi_{\Phi E}$ for all $E \in \mathcal{M}$, and such that the following condition is satisfied for complex-valued $\mu$-measurable functions:

$$
f_{n} \rightarrow f \mu \text {-a.e. on } \Omega \text { implies } \Phi\left(f_{n}\right) \rightarrow \Phi(f) \mu \text {-a.e. on } \Omega \text {. }
$$

Clearly this linear operator $\Phi$ is positive. Further convenient properties which $\Phi$ enjoys as an operator on the complex-valued $\mu$-measurable functions include the following $[16,(4.5)-(4.7)]$ :

$$
\begin{gathered}
\Phi(f g)=\Phi(f) \Phi(g) ; \\
|\Phi f|^{\alpha}=\Phi\left(|f|^{\alpha}\right), \quad \text { for } 0<\alpha<\infty ; \\
\{\omega \in \Omega:(\Phi f)(\omega) \neq 0\}=\Phi\{\omega \in \Omega: f(\omega) \neq 0\} .
\end{gathered}
$$

We shall require the following two results relating separation-preserving operators and $\sigma$-endomorphisms.

(2.9) Proposition ([16, Theorem 4.1]). Suppose that $(\Omega, \mathcal{M}, \mu)$ is a $\sigma$-finite measure space, $1 \leq p<\infty$, and $T: L^{p}(\mu) \rightarrow L^{p}(\mu)$ is a bounded separation-preserving linear operator. For each $E \in \mathcal{M}$ such that $\mu E<\infty$, let

$$
\Phi_{0} E=\left\{\omega \in \Omega:\left(T\left(\chi_{E}\right)\right)(\omega) \neq 0\right\} .
$$

Then $\Phi_{0}$ extends to a unique $\sigma$-endomorphism $\Phi$ of $(\Omega, \mathcal{M}, \mu)$. There is a unique $\mu$-measurable function $h: \Omega \rightarrow \mathbb{C}$ such that $h=0$ a.e. in $\Omega \backslash \Phi(\Omega)$, and, for each $f \in L^{p}(\mu), T f$ is the pointwise product on $\Omega$ of $h$ and $\Phi f$.

(2.10) Proposition ([16, Proposition 4.1]). Suppose that $(\Omega, \mathcal{M}, \mu)$ is a $\sigma$-finite measure space, $1 \leq p<\infty$, and $T$ is a bounded, invertible, separation-preserving linear mapping of $L^{p}(\mu)$ onto $L^{p}(\mu)$. Then, in the notation of Proposition (2.9), the following assertions hold.

(i) For $\mu$-almost all $\omega \in \Omega, h(\omega) \neq 0$.

(ii) $\Phi$ is one-to-one on the space of all complex-valued $\mu$-measurable functions defined on $\Omega$ (hence, as a $\sigma$-endomorphism, $\Phi$ is one-to-one on the class $\mathcal{M}$ of all $\mu$-measurable sets).

(iii) $\Phi$ maps the space of all complex-valued $\mu$-measurable functions defined on $\Omega$ onto itself, and, as a $\sigma$-endomorphism, $\Phi$ maps $\mathcal{M}$ onto $\mathcal{M}$. In particular, $\Phi(\Omega)=\Omega$.

We now assume the hypotheses of Proposition (2.10), and we shall close this section with a few observations. The concomitant notation will remain in effect henceforth. For each $j \in \mathbb{Z}$, it is clear that $\left|T^{j}\right|=|T|^{j}$. Let $\Phi_{j}, h_{j}$ be associated with $T^{j}$ in accordance with Proposition (2.9). Notice that consequently $\Phi_{j}$ and $\left|h_{j}\right|$ 
are associated with $\left|T^{j}\right|$ in Proposition (2.9). Moreover, the Radon-Nikodym Theorem provides a unique sequence $\left\{J_{j}\right\}_{j=-\infty}^{\infty}$ of non-negative, real-valued, measurable functions on $\Omega$ such that for each $j \in \mathbb{Z}$, and each $f \in L^{1}(\mu)$, we have

$$
\int_{\Omega} J_{j}(x)\left(\Phi_{j} f\right)(x) d \mu(x)=\int_{\Omega} f d \mu .
$$

It is now easy to see that for $j \in \mathbb{Z}, k \in \mathbb{Z}$, each of the following relations holds:

$$
\Phi_{j+k}(E)=\Phi_{j}\left(\Phi_{k}(E)\right), \quad \text { for all } E \in \mathcal{M} \text {; }
$$

for each measurable $f: \Omega \rightarrow \mathbb{C}$, we have $\Phi_{j+k}(f)=\Phi_{j}\left(\Phi_{k} f\right), \quad \mu$-a.e.;

$$
\begin{gathered}
h_{j+k}(x)=h_{j}(x)\left(\Phi_{j} h_{k}\right)(x), \quad \text { for } \mu \text {-almost all } x \in \Omega ; \\
J_{j}(x)\left[\Phi_{j}\left(J_{k}\right)\right](x)=J_{j+k}(x), \quad \text { for } \mu \text {-almost all } x \in \Omega .
\end{gathered}
$$

For $j \in \mathbb{Z}$, notice that by Monotone Convergence, (2.11) applies to every real-valued, non-negative, measurable function. In particular, if $\chi_{j}$ denotes the characteristic function of $\left\{x \in \Omega: J_{j}(x)=0\right\}$, then application of $(2.11)$ to $\Phi_{-j}\left(\chi_{j}\right)$ shows, with the aid of (2.13) and (2.10)-(ii), that $J_{j}(x)>0$ for $\mu$-almost all $x \in \Omega$.

\section{The $A_{p}$ Condition and Separation-Preserving Operators}

A bilateral sequence $w=\left\{w_{k}\right\}_{k=-\infty}^{\infty}$ of strictly positive real numbers will be called a weight sequence. If $1 \leq p<\infty$, we shall denote by $\ell^{p}(w)$ the corresponding Banach space consisting of all complex-valued sequences $x=\left\{x_{k}\right\}_{k=-\infty}^{\infty}$ such that

$$
\|x\|_{\ell^{p}(w)} \equiv\left\{\sum_{k=-\infty}^{\infty}\left|x_{k}\right|^{p} w_{k}\right\}^{1 / p}<\infty .
$$

(3.1) Definition ([15]). Suppose that $1<p<\infty$, and $C$ is a non-negative real number. A weight sequence $w$ is said to satisfy the $A_{p}$ condition (with constant $C$ ) provided that whenever $K \in \mathbb{Z}, L \in \mathbb{Z}$, and $K \leq L$, we have

$$
\left[\sum_{k=K}^{L} w_{k}\right]\left[\sum_{k=K}^{L}\left(w_{k}\right)^{-1 /(p-1)}\right]^{p-1} \leq C(L-K+1)^{p} .
$$

(In particular, it follows that $C \geq 1$.)

The $A_{p}$ condition was originally introduced in continuous variable form by $\mathrm{B}$. Muckenhoupt [23] in order to characterize the weights in $\mathbb{R}^{n}$ for which the HardyLittlewood maximal operator is of strong type $(p, p)$, if $1<p<\infty$ [23, Theorem 9]. Analogous results for the Hilbert transform in the setting of $\mathbb{R}$, as well as in the periodic and discrete cases, were obtained in [15]. Weighted norm inequalities for the classical operators of Fourier analysis have been featured extensively in recent years (see, for instance, [13], [17]). In Definition (3.1) we have used the discrete version of the $A_{p}$ condition because of its intimate relationship with separationpreserving operators, as established in [21] and generalized in [22]. More precisely, we shall rely on the theorem of $[21](=[22$, Theorem $(2.4)])$, which takes the following form when stated for separation-preserving operators. 
(3.2) Theorem. Suppose that $(\Omega, \mathcal{M}, \mu)$ is a $\sigma$-finite measure space, $1<p<\infty$, and $T$ is a bounded, invertible, separation-preserving linear mapping of $L^{p}(\mu)$ onto $L^{p}(\mu)$. The following assertions are equivalent.

(i) There is a real constant $C>0$ such that for any $f \in L^{p}(\mu)$,

$$
\int_{\Omega}|M f|^{p} d \mu \leq C \int_{\Omega}|f|^{p} d \mu
$$

where $M$ is the maximal operator defined on $L^{p}(\mu)$ by

$$
\begin{aligned}
& M f=\sup _{n \geq 0} \frac{1}{2 n+1} \sum_{j=-n}^{n}\left|T^{j} f\right| . \\
& \mathfrak{s} \equiv \sup _{n \geq 0}\left\|\frac{1}{2 n+1} \sum_{j=-n}^{n}|T|^{j}\right\|<\infty .
\end{aligned}
$$

(iii) There is a real number $C>0$ such that for $\mu$-almost all $x \in \Omega$, the weight sequence $\left\{\left|h_{k}(x)\right|^{-p} J_{k}(x)\right\}_{k=-\infty}^{\infty}$ satisfies the $A_{p}$ condition with constant $C$.

Remarks. The reasoning in [21, pp. 147-148] shows that when the hypotheses and equivalent conditions listed in Theorem (3.2) are satisfied, the constant $C$ in (3.2)(iii) can be chosen so as to depend only on $p$ and the number $\mathfrak{s}$ in (3.2)-(ii).

With the aid of the methods in [21] (especially, the equivalence of (3.2)-(ii) and (3.2)-(iii)), the boundedness properties of the discrete Hilbert transform in the presence of the $A_{p}$ condition [15, Theorem 10], and simulated transference techniques, R. Sato [24] established a maximal theorem for the ergodic Hilbert transform defined by an operator $T$ which satisfies the hypotheses and equivalent conditions listed in Theorem (3.2). For purposes of the spectral theory which will play a crucial rôle in the subsequent sections, it is convenenient to observe here that if such an operator $T$ is replaced by $e^{i t} T$, where $t \in[0,2 \pi]$, then the reasoning and estimates of [24] are readily seen to hold uniformly in $t$. We state the outcome in the following theorem.

(3.3) Theorem. Suppose that $(\Omega, \mathcal{M}, \mu)$ is a $\sigma$-finite measure space, $1<p<\infty$, and $T$ is a bounded, invertible, separation-preserving linear mapping of $L^{p}(\mu)$ onto $L^{p}(\mu)$ such that

$$
\sup _{n \geq 0}\left\|\frac{1}{2 n+1} \sum_{j=-n}^{n}|T|^{j}\right\|<\infty .
$$

Then there is a real constant $C>0$ such that for all $t \in[0,2 \pi]$, and all $f \in L^{p}(\mu)$,

$$
\int_{\Omega}\left[\sup _{n \geq 1}\left|\sum_{0<|k| \leq n} \frac{1}{k} e^{i k t} T^{k} f\right|\right]^{p} d \mu \leq C \int_{\Omega}|f|^{p} d \mu .
$$

Moreover, for each $t \in[0,2 \pi]$, and each $f \in L^{p}(\mu)$, the sequence

$$
\left\{\sum_{0<|k| \leq n} \frac{1}{k} e^{i k t} T^{k} f\right\}_{n=1}^{\infty}
$$

converges $\mu$-a.e. on $\Omega$, and in the norm topology of $L^{p}(\mu)$. 


\section{Separation-Preserving Operators and Spectral Decomposability}

In this section, we show that an operator satisfying the hypotheses of Theorem (3.3) can be written as a spectral integral analogous in form to that representing a unitary operator. We then describe a structural consequence needed in the next section in order to develop a Littlewood-Paley theory for separation-preserving operators. We first take up some background items from spectral theory, starting with the relevant notion for spectral decomposability.

Definition. Let $\mathfrak{B}(Y)$ denote the Banach algebra of all bounded linear mappings of a Banach space $Y$ into itself, and let $I$ be the identity operator on $Y$. A spectral family in $Y$ is a projection-valued function $F(\cdot)$ mapping the real line $\mathbb{R}$ into $\mathfrak{B}(Y)$, and having the following properties:

(i) $\sup \{\|F(\lambda)\|: \lambda \in \mathbb{R}\}<\infty$;

(ii) $F(\lambda) F(\tau)=F(\tau) F(\lambda)=F(\lambda)$ whenever $\lambda \leq \tau$;

(iii) $F(\cdot)$ is right-continuous on $\mathbb{R}$ with respect to the strong operator topology of $\mathfrak{B}(Y)$;

(iv) at each $\lambda \in \mathbb{R}, F(\cdot)$ has a left-hand limit $F\left(\lambda^{-}\right)$in the strong operator topology of $\mathfrak{B}(Y)$;

(v) with respect to the strong operator topology of $\mathfrak{B}(Y), F(\lambda) \rightarrow I$ as $\lambda \rightarrow+\infty$, and $F(\lambda) \rightarrow 0$ as $\lambda \rightarrow-\infty$.

If there is a compact interval $[a, b]$ such that $F(\lambda)=0$ for $\lambda<a$ and $F(\lambda)=I$ for $\lambda \geq b$, then we say that $F(\cdot)$ is concentrated on $[a, b]$.

Corresponding to any spectral family $F(\cdot)$ of projections in $Y$, a RiemannStieltjes notion of spectral integration with respect to $F(\cdot)$ can be defined as follows. For convenience, we suppose here that $F(\cdot)$ is concentrated on a compact interval $K=[a, b]$ of $\mathbb{R}$. Given a bounded function $f: K \rightarrow \mathbb{C}$, for each partition $\mathcal{P}=\left(\lambda_{0}, \lambda_{1}, \ldots, \lambda_{n}\right)$ of $K$ we put

$$
\mathcal{S}(\mathcal{P} ; f, F)=\sum_{k=1}^{n} f\left(\lambda_{k}\right)\left\{F\left(\lambda_{k}\right)-F\left(\lambda_{k-1}\right)\right\} .
$$

If the net $\{\mathcal{S}(\mathcal{P} ; f, F)\}$ converges in the strong operator topology of $\mathfrak{B}(Y)$ as $\mathcal{P}$ increases through the partitions of $K$ directed by inclusion, then the strong limit is called the spectral integral of $f$ with respect to $F(\cdot)$, and denoted by $\int_{[a, b]} f d F$. We then further define $\int_{[a, b]}^{\oplus} f d F$ by writing

$$
\int_{[a, b]}^{\oplus} f d F=f(a) F(a)+\int_{[a, b]} f d F .
$$

A standard feature of this notion of spectral integration is that $\int_{[a, b]} f d F$ exists whenever $f \in B V([a, b])$ (see, for example, the simplified account in [6, §2]). Moreover the mapping $f \in B V([a, b]) \rightarrow \int_{[a, b]}^{\oplus} f d F$ is an identity-preserving algebra homomorphism of $B V([a, b])$ into $\mathfrak{B}(Y)$ such that

$$
\left\|\int_{[a, b]}^{\oplus} f d F\right\| \leq\|f\|_{K} \sup \{\|F(\lambda)\|: \lambda \in \mathbb{R}\}, \quad \text { for all } f \in B V([a, b]),
$$

where $\|\cdot\|_{K}$ denotes the Banach algebra norm of $B V([a, b])$ specified by

$$
\|f\|_{K}=|f(b)|+\operatorname{var}(f,[a, b]) .
$$


A precise abstract definition of the operators having a "unitary-like" spectral representation can now be given as follows.

Definition. An operator $U \in \mathfrak{B}(Y)$ is said to be trigonometrically well-bounded provided there is a spectral family $E(\cdot)$ in $Y$ concentrated on $[0,2 \pi]$ such that $U=\int_{[0,2 \pi]}^{\oplus} e^{i \lambda} d E(\lambda)$. In this case it is always possible to arrange matters so that we also have $E\left((2 \pi)^{-}\right)=I$. With this additional property the spectral family $E(\cdot)$ is uniquely determined by $U$, and called the spectral decomposition of $U$.

Trigonometrically well-bounded operators were introduced in [4], to which we refer the reader for basic details. Some key relationships between trigonometrically well-bounded operators and Fourier series methods were established in [5]. In particular, [5, Corollary (2.10)] provides the following sufficient condition for an operator to be trigonometrically well-bounded.

(4.1) Proposition. Let $X$ be a reflexive Banach space, and let $U \in \mathfrak{B}(X)$ be invertible. For each positive integer $n$ and each $t \in[0,2 \pi]$, put

$$
s_{n}(U, t)=\sum_{0<|k| \leq n} \frac{1}{k} e^{i k t} U^{k} .
$$

If

$$
\rho \equiv \sup \left\{\left\|s_{n}(U, t)\right\|: n \geq 1, t \in[0,2 \pi]\right\}<\infty,
$$

then $U$ is trigonometrically well-bounded, and the spectral decomposition $E(\cdot)$ of $U$ satisfies

$$
\sup \{\|E(\lambda)\|: \lambda \in \mathbb{R}\} \leq 3\left\{1+(2 \pi)^{-1} \rho\right\} .
$$

Comparison of Theorems (3.3) and (4.1) provides the following result.

(4.2) Theorem. Suppose that $(\Omega, \mathcal{M}, \mu)$ is a $\sigma$-finite measure space, $1<p<\infty$, and $T$ is a bounded, invertible, separation-preserving linear mapping of $L^{p}(\mu)$ onto $L^{p}(\mu)$ such that

$$
\mathfrak{s} \equiv \sup _{n \geq 0}\left\|\frac{1}{2 n+1} \sum_{j=-n}^{n}|T|^{j}\right\|<\infty .
$$

Then $T$ is trigonometrically well-bounded.

Remarks. With due attention to a few extra details, it is not difficult to see that under the hypotheses of Theorem (4.2) there is a positive real constant $\delta$, depending only on $p$ and $\mathfrak{s}$, such that the spectral decomposition $E(\cdot)$ of $T$ satisfies

$$
\|E(\lambda)\| \leq \delta, \quad \text { for all } \lambda \in \mathbb{R}
$$

We shall now sketch some examples to illustrate Theorem (4.2) in the absence of power-boundedness. As remarked earlier, the $A_{p}$ condition was originally introduced in the continuous variable case [23] in order to characterize the strong boundedness of the Hardy-Littlewood maximal operator in the setting of weighted norm inequalities. Easy changes in the proofs in [23] give a similar result for the integers (see [21, §2]-for corresponding results regarding the one-sided HardyLittlewood maximal operator, see [25] and [22, p. 70]). For our immediate purposes the following discrete result, included in $[21, \S 2]$, will suffice. 
(4.3) Scholium. Suppose that $1<p<\infty$, and $w=\left\{w_{k}\right\}_{k=-\infty}^{\infty}$ is a weight sequence which satisfies the $A_{p}$ condition (3.1). For each $f: \mathbb{Z} \rightarrow \mathbb{C}$, define $f^{*}$ pointwise on $\mathbb{Z}$ by writing

$$
f^{*}(j)=\sup _{n \geq 0} \frac{1}{2 n+1} \sum_{k=-n}^{n}|f(j-k)| .
$$

Then there is a real constant $C>0$ such that for all $f: \mathbb{Z} \rightarrow \mathbb{C}$ we have

$$
\sum_{j=-\infty}^{\infty}\left|f^{*}(j)\right|^{p} w_{j} \leq C \sum_{j=-\infty}^{\infty}|f(j)|^{p} w_{j} .
$$

It follows that under the hypotheses of Scholium (4.3), the right bilateral shift $U$ defined on $\ell^{p}(w)$ by $U\left(\left\{x_{k}\right\}_{k=-\infty}^{\infty}\right)=\left\{x_{k-1}\right\}_{k=-\infty}^{\infty}$ is a bounded invertible operator having the left bilateral shift on $\ell^{p}(w)$ as inverse: $U^{-1}\left(\left\{x_{k}\right\}_{k=-\infty}^{\infty}\right)=\left\{x_{k+1}\right\}_{k=-\infty}^{\infty}$. Obviously $U$ and $U^{-1}$ are positivity-preserving, and for each $j \in \mathbb{Z}, k \in \mathbb{Z}, x \in$ $\ell^{p}(w)$, we have $\left(U^{k} x\right)(j)=x_{j-k}$. Another application of Scholium (4.3) now shows at once that $U$ satisfies the hypotheses on $T$ of Theorem (4.2), with $\Omega=\mathbb{Z}$, and $\mu$ the measure which assigns to each integer $k$ the mass $w_{k}$. (This can also be seen by using (3.1) to infer directly that $U$ satisfies (3.2)-(iii).) Hence the right bilateral shift $\mathrm{U}$ is trigonometrically well-bounded on $\ell^{p}(w)-$ a result obtained by other reasoning in [7, Theorem (4.2)]. In particular, if we consider the weight sequence $w$ specified by taking $w_{0}=1$ and $w_{k}=|k|^{\alpha}$, for $k \neq 0$, where $\alpha$ is a constant satisfying $0<\alpha<1$, then straightforward reasoning based on [13, p. 407] shows that $w$ satisfies the $A_{p}$ condition provided that $1+\alpha<p$. Under these particular circumstances, it is easy to see that for all $n \in \mathbb{Z},\left\|U^{n}\right\|=(1+|n|)^{\alpha / p}$, and consequently $\sup _{n \in \mathbb{Z}}\left\|U^{n}\right\|=\infty$.

For the construction of a similar example in the setting of $\mathbb{R}$, we again suppose that $0<\alpha<1$, and $1+\alpha<p<\infty$. Define the weight function $\mathfrak{W}$ on $\mathbb{R}$ by putting $\mathfrak{W}(x) \equiv|x|^{\alpha}+1$, and let $\mu$ be the $\sigma$-finite measure on $\mathbb{R}$ specified by $d \mu=\mathfrak{W}(x) d x$. Elementary arguments show that the translation operator $\mathfrak{U}$ defined by writing $(\mathfrak{U} f)(x)=f(x+1)$ is a bounded, invertible, positivity-preserving linear mapping of $L^{p}(\mu)$ onto $L^{p}(\mu)$ such that $\mathfrak{U}^{-1}$ is positivity-preserving, and such that for each $n \in \mathbb{Z},\left\|\mathfrak{U}^{n}\right\|^{p}=\sup _{x \in \mathbb{R}} \frac{\mathfrak{W}(x)}{\mathfrak{W}(x+n)} \geq \mathfrak{W}(-n)$. Consequently, $\left\|\mathfrak{U}^{n}\right\| \rightarrow \infty$ as $|n| \rightarrow \infty$. Since $\mathfrak{W}$ is an $A_{p}$ weight in $\mathbb{R}$, it is not difficult to see that $\mathfrak{U}$ satisfies the condition stated in Theorem (3.2)-(iii), and consequently $\mathfrak{U}$ satisfies the hypotheses of Theorem (4.2) without being power-bounded.

In order to obtain a broad class of operators which satisfy the hypotheses of Theorem (4.2) without being power-bounded, one can adapt the reasoning for flows in $[20, \S 6]$, while making use of Theorem (3.2) above. The following single operator version of [20, Theorem 7] results.

Proposition. Suppose that $1<p<\infty,(\Omega, \mathcal{M}, \mu)$ is a non-atomic measure space such that $0<\mu(\Omega)<\infty$, and $\tau$ is a one-to-one mapping of $\Omega$ onto $\Omega$ which is measure-preserving and ergodic for $(\Omega, \mathcal{M}, \mu)$. Then there is a finite measure $\nu$ defined on $\mathcal{M}$ and equivalent to $\mu$ such that the composition mapping $T: g \in$ $L^{p}(\nu) \mapsto g \circ \tau$ is a positivity-preserving linear operator satisfying the hypotheses of Theorem (4.2) on $L^{p}(\nu)$ and having the property that $\sup _{n \in \mathbb{Z}}\left\|T^{n}\right\|=\infty$. 
We close this section with a result regarding the Fourier analysis of separationpreserving operators. For a function $\phi \in B V(\mathbb{T})$, denote the Fourier transform of $\phi$ by $\widehat{\phi}$,

$$
\widehat{\phi}(k) \equiv(2 \pi)^{-1} \int_{0}^{2 \pi} \phi\left(e^{i t}\right) e^{-i k t} d t,
$$

and define $\phi^{\ddagger}: \mathbb{R} \rightarrow \mathbb{C}$ by writing

$$
\phi^{\ddagger}(t)=\frac{1}{2}\left\{\lim _{s \rightarrow t+} \phi\left(e^{i s}\right)+\lim _{s \rightarrow t-} \phi\left(e^{i s}\right)\right\} .
$$

Notice that $\phi^{\ddagger}$ is periodic of period $2 \pi$, and has bounded variation on each compact interval of $\mathbb{R}$. Combining [6, Theorem (3.10)-(i)] with Theorem (4.2), we obtain the following theorem.

(4.5) Theorem. Assume the hypotheses of Theorem (4.2), and let $E(\cdot)$ be the spectral decomposition of $T$. Then for each $\phi \in B V(\mathbb{T})$, the series $\sum_{k=-\infty}^{\infty} \widehat{\phi}(k) T^{k}$ is $(C, 1)$-summable in the strong operator topology of $\mathfrak{B}\left(L^{p}(\mu)\right)$ to $\int_{[0,2 \pi]}^{\oplus} \phi^{\ddagger}(\lambda) d E(\lambda)$, that is,

$$
\left\|\left\{\sum_{k=-n}^{n}\left(1-\frac{|k|}{n+1}\right) \widehat{\phi}(k) T^{k}\right\} f-\left\{\int_{[0,2 \pi]}^{\oplus} \phi^{\ddagger}(\lambda) d E(\lambda)\right\} f\right\|_{L^{p}(\mu)} \rightarrow 0,
$$

as $n \rightarrow \infty$,

for each $f \in L^{p}(\mu)$.

\section{Littlewood-Paley Theory for Separation-Preserving Operators}

Our goal in this section is to show that an operator $T$ satisfying the hypotheses of Theorem (4.2) provides $L^{p}(\mu)$ with an abstract analogue of the classical Littlewood-Paley Theorem for $\ell^{p}(\mathbb{Z})$ ([12, Theorem 7.2.1]). Because of the central rôle played in our considerations by the $A_{p}$ condition figuring in Theorem (3.2)-(iii), we shall proceed from a known version of the Marcinkiewicz Multiplier Theorem for weighted sequence spaces. In order to formulate this version and to fix some essential notation, we begin by recalling the dyadic decomposition of $\mathbb{T}$, which is described in the following manner. For $j \in \mathbb{Z}$, let $t_{j}$ be the $j^{\underline{t h}}$ dyadic point of $(0,2 \pi)$ specified by

$$
t_{j}= \begin{cases}2^{j-1} \pi, & \text { if } j \leq 0 \\ 2 \pi-2^{-j} \pi, & \text { if } j>0\end{cases}
$$

and put

$$
\omega_{j}=e^{i t_{j}} \quad \text { for } j \in \mathbb{Z} .
$$

Also, for $j \in \mathbb{Z}$, let $\Gamma_{j}$ be the arc specified by

$$
\Gamma_{j}=\left\{e^{i t}: t_{j}<t<t_{j+1}\right\},
$$

and denote the closure in $\mathbb{T}$ of $\Gamma_{j}$ by $\Delta_{j}$. With this notation, the functions $\phi$ mapping $\mathbb{T}$ into the complex numbers $\mathbb{C}$ which satisfy the hypotheses of the classical Strong Marcinkiewicz Multiplier Theorem for $\ell^{p}(\mathbb{Z})$ can be characterized by the condition

$$
\|\phi\|_{\mathfrak{M}(\mathbb{T})} \equiv \sup \{|\phi(z)|: z \in \mathbb{T}\}+\sup \left\{\operatorname{var}\left(\phi, \Delta_{j}\right): j \in \mathbb{Z}\right\}<\infty .
$$


We shall call the functions satisfying this condition Marcinkiewicz multipliers, and shall denote the set of all Marcinkiewicz multipliers by $\mathfrak{M}(\mathbb{T})$. Notice that with pointwise operations and the norm $\|\cdot\|_{\mathfrak{M}(\mathbb{T})}, \mathfrak{M}(\mathbb{T})$ is a Banach algebra.

Given a function $\psi \in L^{\infty}(\mathbb{T})$, we shall symbolize the inverse Fourier transform of $\psi$ by $\psi^{\vee}$ : for all $k \in \mathbb{Z}, \psi^{\vee}(k)=(2 \pi)^{-1} \int_{0}^{2 \pi} \psi\left(e^{i t}\right) e^{i k t} d t$. If $1<p<\infty$, and $w=\left\{w_{k}\right\}_{k=-\infty}^{\infty}$ is a weight sequence, we shall say that $\psi \in L^{\infty}(\mathbb{T})$ is a multiplier for $\ell^{p}(w)$ provided that: (i) for each $y=\left\{y_{k}\right\}_{k=-\infty}^{\infty} \in \ell^{p}(w)$, and each $j \in \mathbb{Z}$, the series $\left(\psi^{\vee} * y\right)(j) \equiv \sum_{k=-\infty}^{\infty} \psi^{\vee}(j-k) y_{k}$ converges absolutely, and (ii) the mapping $S_{\psi}: y \in \ell^{p}(w) \rightarrow \psi^{\vee} * y$ is a bounded linear mapping of $\ell^{p}(w)$ into $\ell^{p}(w)$. (Since $p$ and $w$ will always be clear from the context, this notation suppresses the dependence of $S_{\psi}$ on them.) The requisite weighted Marcinkiewicz Multiplier Theorem for $\mathbb{Z}$, which is essentially due to D.S. Kurtz [17], can now be stated as follows.

(5.1) Theorem. Suppose that $1<p<\infty, w$ is a weight sequence satisfying the $A_{p}$ condition (3.1) with constant $C$, and $\phi \in \mathfrak{M}(\mathbb{T})$. Then $\phi$ is a multiplier for $\ell^{p}(w)$, and the corresponding operator $S_{\phi}$ on $\ell^{p}(w)$ satisfies

$$
\left\|S_{\phi}\right\| \leq K\|\phi\|_{\mathfrak{M}(\mathbb{T})},
$$

where $K>0$ is a real constant depending only on $p$ and $C$.

Remarks. (i) Notice that in the special case where $w_{k}=1$ for all $k \in \mathbb{Z}$, the foregoing definition of a multiplier for $\ell^{p}(w)$ coincides with the classical notion of a Fourier multiplier for $\ell^{p}(\mathbb{Z})$. (ii) The counterpart for $\mathbb{R}$ of the statement in Theorem (5.1) was established by Kurtz in [17, proof of Theorem 2]. Since a weight sequence satisfying the $A_{p}$ condition gives rise naturally to an $A_{p}$ weight on $\mathbb{R}[15$, proof of Theorem 10], Kurtz's result can be transplanted to $\mathbb{Z}$ in the form of Theorem (5.1) by methods like those used in [1, proof of Theorem 1$]$. We omit the details for expository reasons.

In order to use Theorem (5.1) in conjunction with the Cesàro averages appearing in the conclusion of Theorem (4.5), we shall require the following analogue of the classical convolution theorem for Fourier multipliers.

(5.2) Theorem. Suppose that $1<p<\infty, w$ is a weight sequence, $\psi$ is a multiplier for $\ell^{p}(w)$, and $\mathfrak{k} \in L^{1}(\mathbb{T})$. Then the convolution $\mathfrak{k} * \psi$ is a multiplier for $\ell^{p}(w)$, and

$$
\left\|S_{\mathfrak{k} * \psi}\right\| \leq\|\mathfrak{k}\|_{L^{1}(\mathbb{T})}\left\|S_{\psi}\right\| .
$$

Proof. For each $z \in \mathbb{T}$, denote the corresponding character of $\mathbb{Z}$ by $\gamma_{z}$, and let $\lambda$ designate normalized Haar measure on $\mathbb{T}$. Using Bochner integration, we define the linear operator $\mathcal{W}: \ell^{p}(w) \rightarrow \ell^{p}(w)$ as follows:

$$
\mathcal{W} y=\int_{\mathbb{T}} \mathfrak{k}(z)\left[\gamma_{z}\left(S_{\psi}\left(\overline{\gamma_{z}} y\right)\right)\right] d \lambda(z), \quad \text { for all } y \in \ell^{p}(w) .
$$

It is clear that $\mathcal{W}$ is a bounded operator on $\ell^{p}(w)$, with

$$
\|\mathcal{W}\| \leq\|\mathfrak{k}\|_{L^{1}(\mathbb{T})}\left\|S_{\psi}\right\|
$$

Since point evaluation at each integer is a continuous linear functional on $\ell^{p}(w)$, the required conclusions are readily obtained by applying the point evaluations to (5.3) in order to infer that $\mathcal{W}=S_{\mathfrak{k} * \psi}$. 
Let $\Sigma_{d}$ denote the sigma-algebra of subsets of $\mathbb{T}$ generated by the class $\mathcal{D}_{\mathbb{T}}$ consisting of the sets $\Gamma_{j}(j \in \mathbb{Z})$ together with the singleton sets $\left\{\omega_{j}\right\}(j \in \mathbb{Z})$ and $\{1\}$. This dyadic sigma-algebra $\Sigma_{d}$ has the following obvious description.

(5.4) For each $\sigma \in \Sigma_{d}$, there is a unique subclass $\mathcal{A}_{\sigma}$ of $\mathcal{D}_{\mathbb{T}}$ such that

$$
\sigma=\bigcup\left\{\alpha: \alpha \in \mathcal{A}_{\sigma}\right\}
$$

We now assume the hypotheses of Theorem (4.2), and we shall show that the spectral decomposition $E(\cdot)$ of $T$ gives rise to a strongly countably additive spectral measure on $\Sigma_{d}$. The preliminary step is to define the projection-valued function $\mathcal{E}_{0}(\cdot)$ on $\mathcal{D}_{\mathbb{T}}$ as follows.

Definition. For each $j \in \mathbb{Z}$, define $\mathcal{E}_{0}\left(\Gamma_{j}\right)$ by putting

$$
\mathcal{E}_{0}\left(\Gamma_{j}\right)=E\left(t_{j+1}^{-}\right)-E\left(t_{j}\right)
$$

and let

$$
\mathcal{E}_{0}(\{1\})=E(0) ; \quad \mathcal{E}_{0}\left(\left\{\omega_{j}\right\}\right)=E\left(t_{j}\right)-E\left(t_{j}^{-}\right), \quad \text { for } j \in \mathbb{Z} .
$$

As will be seen shortly, the following lemma constitutes the key step in establishing the existence of the dyadic spectral measure induced by $E(\cdot)$. Notice first that if $\alpha \in \mathcal{D}_{\mathbb{T}}, \beta \in \mathcal{D}_{\mathbb{T}}$, and $\alpha \neq \beta$, then $\mathcal{E}_{0}(\alpha) \mathcal{E}_{0}(\beta)=\mathcal{E}_{0}(\beta) \mathcal{E}_{0}(\alpha)=0$.

(5.5) Lemma. Let $\mathfrak{F}$ be the class consisting of all $\sigma \in \Sigma_{d}$ such that (in the notation of (5.4)) $\mathcal{A}_{\sigma}$ is finite. Extend $\mathcal{E}_{0}$ to the projection-valued function $\mathcal{E}_{1}$ defined on $\mathfrak{F}$ by writing, for each $\sigma \in \mathfrak{F}$,

$$
\mathcal{E}_{1}(\sigma)=\sum_{\alpha \in \mathcal{A}_{\sigma}} \mathcal{E}_{0}(\alpha)
$$

Then

$$
\sup \left\{\left\|\mathcal{E}_{1}(\sigma)\right\|: \sigma \in \mathfrak{F}\right\}<\infty
$$

Proof. Let

$$
\mathfrak{F}_{1}=\left\{\sigma \in \mathfrak{F} ; \mathcal{A}_{\sigma} \subseteq\left\{\Gamma_{j}: j \in \mathbb{Z}\right\}\right\}, \quad \mathfrak{F}_{2}=\left\{\sigma \in \mathfrak{F}: \mathcal{A}_{\sigma} \subseteq\left\{\left\{\omega_{j}\right\}: j \in \mathbb{Z}\right\}\right\} .
$$

It clearly suffices to show that (5.6) holds for each of $\mathfrak{F}_{1}, \mathfrak{F}_{2}$ in place of $\mathfrak{F}$. For $n \in \mathbb{N}$, let $\kappa_{n}$ denote the Fejér kernel of order $n$ for $\mathbb{T}$, and suppose, to begin with, that $\sigma=\bigcup_{k=1}^{N} \Gamma_{j_{k}}$, where $N \in \mathbb{N}$, and $j_{1}<j_{2}<\cdots<j_{N}$. For $k=1, \cdots, N$, choose $a_{k}, b_{k}$ so that $t_{j_{k}}<a_{k}<b_{k}<t_{j_{k}+1}$. Denote by $\chi$ the characteristic function, defined on $[0,2 \pi]$, of $\bigcup_{k=1}^{N}\left[a_{k}, b_{k}\right]$, and let $\phi \in B V(\mathbb{T})$ be defined by taking $\phi\left(e^{i t}\right)=\chi(t)$, for $0 \leq t \leq 2 \pi$. Obviously $\|\phi\|_{\mathfrak{M}(\mathbb{T})}=3$. It is straightforward to calculate that, in the notation of (4.4)):

$$
\int_{[0,2 \pi]}^{\oplus} \phi^{\ddagger}(t) d E(t)=\sum_{k=1}^{N}\left[\frac{1}{2}\left\{E\left(b_{k}\right)+E\left(b_{k}^{-}\right)\right\}-\frac{1}{2}\left\{E\left(a_{k}\right)+E\left(a_{k}^{-}\right)\right\}\right] .
$$


Let $f \in L^{p}(\mu)$, and temporarily fix $n \in \mathbb{N}$. Then, in terms of the notation established in $\S 2$, we have, for arbitrary $L \in \mathbb{N}$ :

$$
\begin{aligned}
& \int_{\Omega}\left|\sum_{m=-n}^{n} \widehat{\kappa_{n}}(m) \widehat{\phi}(m) T^{m} f\right|^{p} d \mu \\
= & \frac{1}{2 L+1} \int_{\Omega} \sum_{j=-L}^{L}\left|\Phi_{j} \sum_{m=-n}^{n} \kappa_{n}^{\vee}(m) \phi^{\vee}(m) T^{-m} f\right|^{p} J_{j} d \mu \\
= & \frac{1}{2 L+1} \int_{\Omega} \sum_{j=-L}^{L}\left|\Phi_{j} \sum_{m=-n}^{n} \kappa_{n}^{\vee}(m) \phi^{\vee}(m) h_{-m} \Phi_{-m} f\right|^{p} J_{j} d \mu \\
= & \frac{1}{2 L+1} \int_{\Omega} \sum_{j=-L}^{L}\left|\sum_{m=-n}^{n} \kappa_{n}^{\vee}(m) \phi^{\vee}(m) \Phi_{j}\left(h_{-m}\right) \Phi_{j-m} f\right|^{p} J_{j} d \mu \\
= & \frac{1}{2 L+1} \int_{\Omega} \sum_{j=-L}^{L}\left|\sum_{m=-n}^{n} \kappa_{n}^{\vee}(m) \phi^{\vee}(m) h_{j-m} \Phi_{j-m} f\right|^{p} J_{j}\left|h_{j}\right|^{-p} d \mu .
\end{aligned}
$$

From Theorems (3.2)-(iii), (5.1), and (5.2) (the last with $\kappa_{n}$ in place of $\mathfrak{k}$ ), we see that there is a real constant $\eta>0$ (depending only on $p$ and the constant $C$ in (3.2)-(iii)) such that $\mu$-a.e. on $\Omega$ :

$$
\sum_{j=-L}^{L}\left|\sum_{m=-n}^{n} \kappa_{n}^{\vee}(m) \phi^{\vee}(m) h_{j-m} \Phi_{j-m} f\right|^{p} J_{j}\left|h_{j}\right|^{-p} \leq \eta \sum_{j=-n-L}^{n+L}\left|\Phi_{j} f\right|^{p} J_{j} .
$$

Using this in (5.8), we find that

$$
\int_{\Omega}\left|\sum_{m=-n}^{n} \widehat{\kappa_{n}}(m) \widehat{\phi}(m) T^{m} f\right|^{p} d \mu \leq \frac{\eta(2 L+2 n+1)}{2 L+1}\|f\|_{L^{p}(\mu)}^{p} .
$$

Keeping $n$ fixed, and letting $L \rightarrow \infty$, we arrive at the estimate

$$
\int_{\Omega}\left|\sum_{m=-n}^{n} \widehat{\kappa_{n}}(m) \widehat{\phi}(m) T^{m} f\right|^{p} d \mu \leq \eta\|f\|_{L^{p}(\mu)}^{p} .
$$

We now let $n \rightarrow \infty$, and apply Theorem (4.5) together with (5.7) to infer that

$$
\left\|\sum_{k=1}^{N}\left[\frac{1}{2}\left\{E\left(b_{k}\right)+E\left(b_{k}^{-}\right)\right\}-\frac{1}{2}\left\{E\left(a_{k}\right)+E\left(a_{k}^{-}\right)\right\}\right]\right\| \leq \eta^{1 / p} .
$$

We now let $b_{k} \rightarrow t_{j_{k}+1}^{-}$, and $a_{k} \rightarrow t_{j_{k}}^{+}$. This gives $\left\|\mathcal{E}_{1}(\sigma)\right\| \leq \eta^{1 / p}$, and completes the proof for the case when $\sigma \in \mathfrak{F}_{1}$.

Suppose next that $\sigma \in \mathfrak{F}_{2}$, and let $\sigma=\bigcup_{k=1}^{N}\left\{\omega_{j_{k}}\right\}$, where $N \in \mathbb{N}$, and $j_{k_{1}}<$ $j_{k_{2}}<\cdots<j_{k_{N}}$. Choose $\varepsilon>0$ small enough so that the intervals $\left[t_{j_{k}}-\varepsilon, t_{j_{k}}+\varepsilon\right]$, $k=1,2, \cdots, N$, are disjoint, and so that for each $k,\left[t_{j_{k}}-\varepsilon, t_{j_{k}}+\varepsilon\right] \subseteq\left(t_{j_{k}-1}, t_{j_{k}+1}\right)$. Denote by $\chi_{\varepsilon}$ the characteristic function, defined on $[0,2 \pi]$, of $\bigcup_{k=1}^{N}\left[t_{j_{k}}-\varepsilon, t_{j_{k}}+\varepsilon\right]$, and define $\psi \in B V(\mathbb{T})$ by writing $\psi\left(e^{i t}\right)=\chi_{\varepsilon}(t)$, for $0 \leq t \leq 2 \pi$. Notice that $\|\psi\|_{\mathfrak{M}(\mathbb{T})} \leq 3$. The proof of Lemma (5.5) can now be completed by reasoning similar to that for $\mathfrak{F}_{1}$, with $\psi$ used in place of $\phi$.

It is clear from the way in which $\mathcal{E}_{0}$ is defined, together with the properties of the spectral family of projections $E(\cdot)$, that the span of the ranges of the projections 
$\mathcal{E}_{0}(\alpha)$, for $\alpha \in \mathcal{D}_{\mathbb{T}}$, is dense in $L^{p}(\mu)$. It follows immediately from this observation and Lemma (5.5) that for any sequence $\left\{\alpha_{j}\right\}_{j=1}^{\infty}$ of distinct elements of $\mathcal{D}_{\mathbb{T}}$, and for any $f \in L^{p}(\mu)$, the series $\sum_{j=1}^{\infty} \mathcal{E}_{0}\left(\alpha_{j}\right) f$ converges unconditionally in the norm topology of $L^{p}(\mu)$. Hence we can extend $\mathcal{E}_{1}$ to a uniformly bounded projectionvalued function $\mathcal{E}$ defined on $\Sigma_{d}$ by using (5.4) to write

$$
\mathcal{E}(\sigma) f=\sum_{\alpha \in \mathcal{A}_{\sigma}} \mathcal{E}_{0}(\alpha) f, \quad \text { for } \sigma \in \Sigma_{d}, f \in L^{p}(\mu) .
$$

Obviously, for $\sigma \in \Sigma_{d}$, the range of $\mathcal{E}(\sigma)$ is the closed linear span of the ranges of the projections $\mathcal{E}_{0}(\alpha)$, where $\alpha$ runs through $\mathcal{A}_{\sigma}$. Similar reasoning from Lemma (5.5) now shows that $\mathcal{E}$ is strongly countably additive on $\Sigma_{d}$. The remaining properties required of a spectral measure are readily verified for $\mathcal{E}$, and so we summarize the results of this discussion in the following theorem.

(5.10) Theorem. Assume the hypotheses of Theorem (4.2), and let E(.) be the spectral decomposition of $T$. Then the projection-valued function $\mathcal{E}$ defined in (5.9) is a strongly countably additive spectral measure on the dyadic sigma-algebra of $\mathbb{T}$, $\Sigma_{d}$.

A standard procedure for applying Khintchine's Theorem ([19, Theorem 2.b.3]) to spectral measures in Lebesgue spaces now furnishes $L^{p}(\mu)$ with the following abstract analogue of the classical Littlewood-Paley Theorem for $\ell^{p}(\mathbb{Z})$.

(5.11) Corollary. Under the hypotheses of Theorem (5.10), let

$$
\tau=\sup \left\{\|\mathcal{E}(\sigma)\|: \sigma \in \Sigma_{d}\right\}
$$

Then there is a real constant $K_{p}>0$, depending only on $p$, such that whenever $f \in L^{p}(\mu)$, and $\left\{\sigma_{j}\right\}_{j \geq 1}$ is a sequence of mutually disjoint elements of $\Sigma_{d}$ satisfying $\mathbb{T}=\bigcup_{j \geq 1} \sigma_{j}$, we have

$$
K_{p}^{-1} \tau^{-1}\|f\|_{L^{p}(\mu)} \leq\left\|\left\{\sum_{j \geq 1}\left|\mathcal{E}\left(\sigma_{j}\right) f\right|^{2}\right\}^{1 / 2}\right\|_{L^{p}(\mu)} \leq K_{p} \tau\|f\|_{L^{p}(\mu)} .
$$

(5.12) Remark. We observe that Corollary (5.11) reflects and generalizes its classical antecedents by recovering the classical Littlewood-Paley Theorem for $\ell^{p}(\mathbb{Z})$ as a special case. Specifically, when $T$ is the left shift on $\ell^{p}(\mathbb{Z})$, the spectral decomposition $E(\cdot)$ of $T$ is determined as follows (see [14, proof of Theorem 1] or [6, Proposition (4.23)-(ii)]): for each $\lambda \in[0,2 \pi), E(\lambda)$ is the Fourier multiplier transform on $\ell^{p}(\mathbb{Z})$ corresponding to the characteristic function of the arc $\left\{e^{i s}: 0 \leq s \leq \lambda\right\}$. Consequently, it is easy to see that the conclusion of Corollary (5.11) for this special case gives the Littlewood-Paley Theorem for $\ell^{p}(\mathbb{Z})$. More generally, the next item shows that Corollary (5.11) specializes to include the weighted Littlewood-Paley Theorem for $\mathbb{Z}$ (the discrete analogue of the result for $\mathbb{R}$ in [17, Theorem 3.1-(i)]). Notice first that in the setting of Theorem (5.1), the characteristic function $\psi$ of an arbitrary arc of $\mathbb{T}$ is a multiplier for $\ell^{p}(w)$, since $\psi \in B V(\mathbb{T}) \subseteq \mathfrak{M}(\mathbb{T})$. (This property of $\psi$ can be seen more simply as a consequence of the boundedness on $\ell^{p}(w)$ of the discrete Hilbert transform-after direct calculation of $\psi^{\vee}$ or upon application of the weighted version of Stečkin's Theorem [7, Theorem (4.9)].) 
(5.13) Scholium. Suppose that $1<p<\infty$, and $w=\left\{w_{k}\right\}_{k=-\infty}^{\infty}$ is a weight sequence which satisfies the $A_{p}$ condition. Then the left shift $T$ is a trigonometrically well-bounded operator on $\ell^{p}(w)$, and $E(\lambda)=S_{\phi_{\lambda}}$, for $0 \leq \lambda<2 \pi$, where $\phi_{\lambda}$ is the characteristic function defined on $\mathbb{T}$ of the arc $\left\{e^{i s}: 0 \leq s \leq \lambda\right\}$, and $E(\cdot)$ denotes the spectral decomposition of $T$.

Proof. The discussion in $\S 4$ shows that $T^{-1}$ (the right shift on $\ell^{p}(w)$ ) satisfies the hypotheses of Theorem (4.2). Hence so does $T$. Suppose now that $0<u<2 \pi$. Direct calculation shows that, in the notation of Theorem (4.5), we have

$$
\int_{[0,2 \pi]}^{\oplus} \phi_{u}^{\ddagger}(t) d E(t)=\frac{1}{2}\left\{E(u)+E\left(u^{-}\right)-E(0)\right\} .
$$

Since convergence in $\ell^{p}(w)$ implies pointwise convergence on $\mathbb{Z}$, application of Theorem (4.5) to $\phi_{u}$ readily shows that for each $x \in \ell^{p}(w)$,

$$
S_{\phi_{u}} x=\frac{1}{2}\left\{E(u)+E\left(u^{-}\right)-E(0)\right\} x .
$$

Specializing $x$ to be an arbitrary finitely supported sequence $y$, we see that

$$
\left(\phi_{u} \widehat{y}\right)^{\vee}=\frac{1}{2}\left\{E(u)+E\left(u^{-}\right)-E(0)\right\} y .
$$

For $\lambda \in[0,2 \pi)$, we let $u$ in (5.14) run through a strictly decreasing sequence in $(0,2 \pi)$ converging to $\lambda$. Then $\left(\phi_{u} \widehat{y}\right)^{\vee} \rightarrow S_{\phi_{\lambda}} y$ in $\ell^{2}(\mathbb{Z})$, and the right-hand side of (5.14) approaches $\frac{1}{2}\{2 E(\lambda)-E(0)\} y$ in $\ell^{p}(w)$. Consequently,

$$
S_{\phi_{\lambda}}=\frac{1}{2}\{2 E(\lambda)-E(0)\}, \quad \text { for } 0 \leq \lambda<2 \pi .
$$

In particular, by taking $\lambda=0$, we see that $E(0)=0$. Using this in (5.15) completes the proof.

It should be mentioned that the above proof of Scholium (5.13) has relied on the context at hand without involving the abstract theory of trigonometrically wellbounded operators. For example, it follows immediately from [4, Corollary 2.17] that on any Banach space the inverse of a trigonometrically well-bounded operator is trigonometrically well-bounded.

\section{The Abstract Analogue for the Vector Version OF THE M. Riesz THEOREM}

In this section, we use the spectral decomposition $E(\cdot)$ of an operator $T$ satisfying the hypotheses of Theorem (4.2) in order to develop an ergodic counterpart for the Vector-Valued M. Riesz Theorem (see $[12, \S 6.5$, pp. 119-120] for the latter). We begin the discussion with a generalization to square functions of Theorem (5.2).

(6.1) Theorem. Suppose that $1<p<\infty$, w is a weight sequence, $N \in \mathbb{N}, \phi_{j}$ $(1 \leq j \leq N)$ are multipliers for $\ell^{p}(w)$, and $\mathfrak{k} \in L^{1}(\mathbb{T})$. Let $K$ be a constant such that

$$
\begin{aligned}
\left\|\left\{\sum_{j=1}^{N}\left|S_{\phi_{j}} x_{j}\right|^{2}\right\}^{1 / 2}\right\|_{\ell^{p}(w)} \leq K\left\|\left\{\sum_{j=1}^{N}\left|x_{j}\right|^{2}\right\}^{1 / 2}\right\|_{\ell^{p}(w)}, \\
\text { whenever }\left\{x_{j}\right\}_{j=1}^{N} \subseteq \ell^{p}(w) .
\end{aligned}
$$


Then

$$
\begin{aligned}
\left\|\left\{\sum_{j=1}^{N}\left|S_{\mathfrak{k} * \phi_{j}} x_{j}\right|^{2}\right\}^{1 / 2}\right\|_{\ell^{p}(w)} \leq\|\mathfrak{k}\|_{L^{1}(\mathbb{T})} K\left\|\left\{\sum_{j=1}^{N}\left|x_{j}\right|^{2}\right\}^{1 / 2}\right\|_{\ell^{p}(w)}, \\
\text { whenever }\left\{x_{j}\right\}_{j=1}^{N} \subseteq \ell^{p}(w) .
\end{aligned}
$$

Proof. We continue with the notation used in the proof of Theorem (5.2). Suppose that $\left\{x_{j}\right\}_{j=1}^{N} \subseteq \ell^{p}(w)$. For $1 \leq j \leq N, m \in \mathbb{Z}$, we have

$$
\left(S_{\mathfrak{k} * \phi_{j}} x_{j}\right)(m)=\int_{\mathbb{T}} \mathfrak{k}(z) \gamma_{z}(m)\left(S_{\phi_{j}}\left(\overline{\gamma_{z}} x_{j}\right)\right)(m) d \lambda(z) .
$$

Using this, together with the Generalized Minkowski Inequality, we get

$$
\begin{aligned}
& \left\{\sum_{j=1}^{N}\left|\left(S_{\mathfrak{k}_{*} \phi_{j}} x_{j}\right)(m)\right|^{2}\right\}^{1 / 2} \\
= & \left\{\sum_{j=1}^{N}\left|\int_{\mathbb{T}} \mathfrak{k}(z) \gamma_{z}(m)\left(S_{\phi_{j}}\left(\overline{\gamma_{z}} x_{j}\right)\right)(m) d \lambda(z)\right|^{2}\right\}^{1 / 2} \\
\leq & \int_{\mathbb{T}}\left\{\sum_{j=1}^{N}\left|\mathfrak{k}(z)\left(S_{\phi_{j}}\left(\overline{\gamma_{z}} x_{j}\right)\right)(m)\right|^{2}\right\}^{1 / 2} d \lambda(z) .
\end{aligned}
$$

Consequently,

$$
\begin{gathered}
\left\|\left\{\sum_{j=1}^{N}\left|S_{\mathfrak{k} * \phi_{j}} x_{j}\right|^{2}\right\}^{1 / 2}\right\|_{\ell p(w)} \\
\leq\left\{\sum_{m=-\infty}^{\infty} w_{m}\left[\int_{\mathbb{T}}|\mathfrak{k}(z)|\left\{\sum_{j=1}^{N}\left|\left(S_{\phi_{j}}\left(\overline{\gamma_{z}} x_{j}\right)\right)(m)\right|^{2}\right\}^{1 / 2} d \lambda(z)\right]^{p}\right\}^{1 / p} .
\end{gathered}
$$

Another application of the Generalized Minkowski Inequality-this time to the majorant in (6.3)-shows that:

$$
\begin{aligned}
& \left\|\left\{\sum_{j=1}^{N}\left|S_{\mathfrak{k}_{*} \phi_{j}} x_{j}\right|^{2}\right\}^{1 / 2}\right\|_{\ell^{p}(w)} \\
\leq & \int_{\mathbb{T}}|\mathfrak{k}(z)|\left[\sum_{m=-\infty}^{\infty} w_{m}\left\{\sum_{j=1}^{N}\left|\left(S_{\phi_{j}}\left(\overline{\gamma_{z}} x_{j}\right)\right)(m)\right|^{2}\right\}^{p / 2}\right]^{1 / p} d \lambda(z) \\
= & \int_{\mathbb{T}}|\mathfrak{k}(z)|\left\|\left\{\sum_{j=1}^{N}\left|S_{\phi_{j}}\left(\overline{\gamma_{z}} x_{j}\right)\right|^{2}\right\}^{1 / 2}\right\|_{\ell^{p}(w)} d \lambda(z) .
\end{aligned}
$$


From our hypothesis in (6.2), we see that for $z \in \mathbb{T}$ :

$$
\left\|\left\{\sum_{j=1}^{N}\left|S_{\phi_{j}}\left(\overline{\gamma_{z}} x_{j}\right)\right|^{2}\right\}^{1 / 2}\right\|_{\ell^{p}(w)} \leq K\left\|\left\{\sum_{j=1}^{N}\left|x_{j}\right|^{2}\right\}^{1 / 2}\right\|_{\ell^{p}(w)} .
$$

Using this in the majorant of (6.4) completes the proof.

The boundedness of the discrete Hilbert transform on $\ell^{p}(w)$, when $1<p<$ $\infty$ and $w$ is a weight sequence satisfying the $A_{p}$ condition, provides, as in the classical setting of $\ell^{p}(\mathbb{Z})$, the following weighted version of the Vector-Valued M. Riesz Theorem.

(6.5) Theorem. Suppose that $1<p<\infty, w$ is a weight sequence which satisfies the $A_{p}$ condition with a constant $C, N \in \mathbb{N}$, and $\chi_{j}(1 \leq j \leq N)$ are the characteristic functions, defined on $\mathbb{T}$, of arbitrary arcs $\mathfrak{I}_{j}(1 \leq j \leq N)$. Then

$$
\left\|\left\{\sum_{j=1}^{N}\left|S_{\chi_{j}} f_{j}\right|^{2}\right\}^{1 / 2}\right\|_{\ell^{p}(w)} \leq \zeta\left\|\left\{\sum_{j=1}^{N}\left|f_{j}\right|^{2}\right\}^{1 / 2}\right\|_{\ell^{p}(w)},
$$

where $\zeta>0$ is a real constant depending only on $p$ and $C$.

In view of Theorem (6.1), we can infer the following result from Theorem (6.5).

(6.6) Corollary. Assume the hypotheses and notation of Theorem (6.5), and let $\mathfrak{k} \in L^{1}(\mathbb{T})$. Then

$$
\left\|\left\{\sum_{j=1}^{N}\left|S_{\mathfrak{k} * \chi_{j}} f_{j}\right|^{2}\right\}^{1 / 2}\right\|_{\ell^{p}(w)} \leq\|\mathfrak{k}\|_{L^{1}(\mathbb{T})} \zeta\left\|\left\{\sum_{j=1}^{N}\left|f_{j}\right|^{2}\right\}^{1 / 2}\right\|_{\ell^{p}(w)},
$$

whenever $\left\{f_{j}\right\}_{j=1}^{N} \subseteq \ell^{p}(w)$.

The stage is now set for the following ergodic generalization of the Vector-Valued M. Riesz Theorem.

(6.7) Theorem. Let $T$ satisfy the hypotheses of Theorem (4.2), and let $E(\cdot)$ be the spectral decomposition of $T$. Then there is a real constant $K>0$ such that

$$
\left\|\left\{\sum_{j=1}^{\infty}\left|E\left(a_{j}\right) g_{j}\right|^{2}\right\}^{1 / 2}\right\|_{L^{p}(\mu)} \leq K\left\|\left\{\sum_{j=1}^{\infty}\left|g_{j}\right|^{2}\right\}^{1 / 2}\right\|_{L^{p}(\mu)},
$$

for all sequences $\left\{a_{j}\right\}_{j=1}^{\infty} \subseteq[0,2 \pi)$, and all sequences $\left\{g_{j}\right\}_{j=1}^{\infty} \subseteq L^{p}(\mu)$.

Proof. In what follows, the Rademacher averages in Khintchine's Theorem will be expressed in terms of the $N$-fold direct product $D^{N}(N \in \mathbb{N})$ of the multiplicative group $D=\{-1,1\}$. The general element of $D^{N}$ will be written $\epsilon=\left(\epsilon_{1}, \cdots, \epsilon_{N}\right)$, and $d \epsilon$ will be Haar measure on $D^{N}$ normalized to have total mass 1 . Suppose that $\left\{a_{j}\right\}_{j=1}^{\infty} \subseteq[0,2 \pi),\left\{b_{j}\right\}_{j=1}^{\infty} \subseteq(0,2 \pi)$, and $\left\{g_{j}\right\}_{j=1}^{\infty} \subseteq L^{p}(\mu)$. For $j \in \mathbb{N}$, let $\mathfrak{I}_{j}$ be the arc $\left\{e^{i t}: 0 \leq t \leq b_{j}\right\}$, and let $\phi_{j} \in B V(\mathbb{T})$ be the characteristic function of $\mathfrak{I}_{j}$. Suppose that $N \in \mathbb{N}, n \in \mathbb{N}$, and let $\kappa_{n}$ be the Fejér kernel of order $n$ for $\mathbb{T}$. 
By Khintchine's Theorem, we have pointwise on $\Omega$ :

$$
\begin{aligned}
&\left\{\sum_{j=1}^{N}\left|\sum_{m=-n}^{n} \widehat{\kappa_{n}}(m) \widehat{\phi_{j}}(m) T^{m} g_{j}\right|^{2}\right\}^{p / 2} \\
& \leq \mathfrak{C}_{p} \int_{D^{N}}\left|\sum_{j=1}^{N} \epsilon_{j} \sum_{m=-n}^{n}\left(\kappa_{n}\right)^{\vee}(m)\left(\phi_{j}\right)^{\vee}(m) T^{-m} g_{j}\right|^{p} d \epsilon,
\end{aligned}
$$

where, here and henceforth, $\mathfrak{C}_{p}$ denotes a non-negative real constant depending only on $p$ which can change in value from one occurrence to another. Integrating the last inequality with respect to $\mu$ over $\Omega$, we get

$$
\begin{gathered}
\left\|\left\{\sum_{j=1}^{N}\left|\sum_{m=-n}^{n} \widehat{\kappa_{n}}(m) \widehat{\phi_{j}}(m) T^{m} g_{j}\right|^{2}\right\}^{1 / 2}\right\|_{L^{p}(\mu)}^{p} \\
\leq \mathfrak{C}_{p} \int_{D^{N}} \int_{\Omega}\left|\sum_{j=1}^{N} \epsilon_{j} \sum_{m=-n}^{n}\left(\kappa_{n}\right)^{\vee}(m)\left(\phi_{j}\right)^{\vee}(m) T^{-m} g_{j}\right|^{p} d \mu d \epsilon .
\end{gathered}
$$

Hence for arbitrary $L \in \mathbb{N}$, we have

$$
\begin{aligned}
& \left\|\left\{\sum_{j=1}^{N}\left|\sum_{m=-n}^{n} \widehat{\kappa_{n}}(m) \widehat{\phi_{j}}(m) T^{m} g_{j}\right|^{2}\right\}^{1 / 2}\right\|_{L^{p}(\mu)}^{p} \\
\leq & \frac{\mathfrak{C}_{p}}{2 L+1} \int_{D^{N}}\left[\sum_{\nu=-L}^{L} \int_{\Omega} J_{\nu}\left|\Phi_{\nu} \sum_{j=1}^{N} \epsilon_{j} \sum_{m=-n}^{n}\left(\kappa_{n}\right)^{\vee}(m)\left(\phi_{j}\right)^{\vee}(m) h_{-m} \Phi_{-m} g_{j}\right|^{p} d \mu\right] d \epsilon \\
= & \frac{\mathfrak{C}_{p}}{2 L+1} \int_{D^{N}} \int_{\Omega} \sum_{\nu=-L}^{L} J_{\nu}\left|h_{\nu}\right|^{-p}\left|\sum_{j=1}^{N} \epsilon_{j} \sum_{m=-n}^{n}\left(\kappa_{n}\right)^{\vee}(m)\left(\phi_{j}\right)^{\vee}(m) h_{\nu-m} \Phi_{\nu-m} g_{j}\right|^{p} d \mu d \epsilon .
\end{aligned}
$$

Changing the order of integration, we see with the aid of another application of Khintchine's Theorem that

$$
\begin{gathered}
\left\|\left\{\sum_{j=1}^{N}\left|\sum_{m=-n}^{n} \widehat{\kappa_{n}}(m) \widehat{\phi_{j}}(m) T^{m} g_{j}\right|^{2}\right\}^{1 / 2}\right\|_{L^{p}(\mu)}^{p} \\
\leq \frac{\mathfrak{C}_{p}}{2 L+1} \int_{\Omega} \sum_{\nu=-L}^{L} J_{\nu}\left|h_{\nu}\right|^{-p}\left\{\sum_{j=1}^{N}\left|\sum_{m=-n}^{n}\left(\kappa_{n}\right)^{\vee}(m)\left(\phi_{j}\right)^{\vee}(m) h_{\nu-m} \Phi_{\nu-m} g_{j}\right|^{2}\right\}^{p / 2} d \mu .
\end{gathered}
$$


Using (3.2)-(iii) and Corollary (6.6), we observe that $\mu$-a.e. on $\Omega$,

$$
\begin{aligned}
& \sum_{\nu=-L}^{L} J_{\nu}\left|h_{\nu}\right|^{-p}\left\{\sum_{j=1}^{N}\left|\sum_{m=-n}^{n}\left(\kappa_{n}\right)^{\vee}(m)\left(\phi_{j}\right)^{\vee}(m) h_{\nu-m} \Phi_{\nu-m} g_{j}\right|^{2}\right\}^{p / 2} \\
\leq & \zeta^{p} \sum_{\nu=-L-n}^{L+n}\left\{\sum_{j=1}^{N}\left|h_{\nu} \Phi_{\nu}\left(g_{j}\right)\right|^{2}\right\}^{p / 2} J_{\nu}\left|h_{\nu}\right|^{-p} \\
= & \zeta^{p} \sum_{\nu=-L-n}^{L+n} J_{\nu}\left\{\sum_{j=1}^{N}\left|\Phi_{\nu}\left(g_{j}\right)\right|^{2}\right\}^{p / 2},
\end{aligned}
$$

where the constant $\zeta$ depends only on $p$ and the constant $C$ in (3.2)-(iii). Applying this to the majorant in (6.10), we find that

$$
\begin{aligned}
& \left\|\left\{\sum_{j=1}^{N}\left|\sum_{m=-n}^{n} \widehat{\kappa_{n}}(m) \widehat{\phi_{j}}(m) T^{m} g_{j}\right|^{2}\right\}^{1 / 2}\right\|_{L^{p}(\mu)}^{p} \\
\leq & \frac{\mathfrak{C}_{p}}{2 L+1} \zeta^{p} \sum_{\nu=-L-n}^{L+n} \int_{\Omega} J_{\nu} \Phi_{\nu}\left(\left\{\sum_{j=1}^{N}\left|g_{j}\right|^{2}\right\}^{p / 2}\right) d \mu \\
= & \mathfrak{C}_{p} \zeta^{p} \frac{2 L+2 n+1}{2 L+1}\left\|\left\{\sum_{j=1}^{N}\left|g_{j}\right|^{2}\right\}^{1 / 2}\right\|_{L^{p}(\mu)}^{p} .
\end{aligned}
$$

Letting $L \rightarrow \infty$ in (6.11), we infer that

$$
\left\|\left\{\sum_{j=1}^{N}\left|\sum_{m=-n}^{n} \widehat{\kappa_{n}}(m) \widehat{\phi_{j}}(m) T^{m} g_{j}\right|^{2}\right\}^{1 / 2}\right\|\left\|_{L^{p}(\mu)} \leq \mathfrak{C}_{p} \zeta\right\|\left\{\sum_{j=1}^{N}\left|g_{j}\right|^{2}\right\}^{1 / 2} \|_{L^{p}(\mu)} .
$$

Next we let $n \rightarrow \infty$ in (6.12), and apply Theorem (4.5) to deduce that

$$
\left\|\left\{\sum_{j=1}^{N}\left|\int_{0,2 \pi]}^{\oplus} \phi_{j}^{\ddagger} d E g_{j}\right|^{2}\right\}^{1 / 2}\right\|_{L^{p}(\mu)} \leq \mathfrak{C}_{p} \zeta\left\|\left\{\sum_{j=1}^{N}\left|g_{j}\right|^{2}\right\}^{1 / 2}\right\|_{L^{p}(\mu)} .
$$

It is easy to see directly that for $1 \leq j \leq N$,

$$
\int_{[0,2 \pi]}^{\oplus} \phi_{j}^{\ddagger} d E=\frac{1}{2}\left\{E\left(b_{j}\right)+E\left(b_{j}^{-}\right)\right\}-\frac{1}{2} E(0) .
$$

Using this in (6.13), and then letting $b_{j} \rightarrow a_{j}^{+}$, for $1 \leq j \leq N$, we arrive at

$$
\left\|\left\{\sum_{j=1}^{N}\left|\left(E\left(a_{j}\right)-\frac{1}{2} E(0)\right) g_{j}\right|^{2}\right\}^{1 / 2}\right\|_{L^{p}(\mu)} \leq \mathfrak{C}_{p} \zeta\left\|\left\{\sum_{j=1}^{N}\left|g_{j}\right|^{2}\right\}^{1 / 2}\right\|_{L^{p}(\mu)} .
$$


Hence by the Marcinkiewicz-Zygmund Inequality [12, pp. 203-204], we see that

$$
\left\|\left\{\sum_{j=1}^{N}\left|E\left(a_{j}\right) g_{j}\right|^{2}\right\}^{1 / 2}\right\|_{L^{p}(\mu)} \leq\left(\mathfrak{C}_{p} \zeta+\frac{\|E(0)\|}{2}\right)\left\|\left\{\sum_{j=1}^{\infty}\left|g_{j}\right|^{2}\right\}^{1 / 2}\right\|_{L^{p}(\mu)} .
$$

The proof can now be completed by letting $N \rightarrow \infty$ in (6.14).

(6.15) Remark. We observe that in the setting of Remark (5.12), the conclusion of Theorem (6.7) is equivalent to the square function estimate asserted by the VectorValued M. Riesz Theorem for $\ell^{p}(\mathbb{Z})[12, \S 6.5$, pp. 119-120].

\section{REFERENCES}

[1] P. Auscher and M.J. Carro, On relations between operators on $\mathbb{R}^{N}, \mathbb{T}^{N}$ and $\mathbb{Z}^{N}$, Studia Math. 101 (1992), 165-182. MR 94b:42007

[2] S. Banach, Théorie des Opérations Linéaires, Monografje Matematyczne, Tom I, Warsaw, 1932.

[3] E. Berkson, J. Bourgain, and T.A. Gillespie, On the almost everywhere convergence of ergodic averages for power-bounded operators on $L^{p}$-subspaces, Integral Equations and Operator Theory 14 (1991), 678-715. MR 92j:47024

[4] E. Berkson and T.A. Gillespie, AC functions on the circle and spectral families, J. Operator Theory 13 (1985), 33-47. MR 86c: 47045

[5] E. Berkson and T.A. Gillespie, Fourier series criteria for operator decomposability, Integral Equations and Operator Theory 9 (1986), 767-789. MR 87m:47082

[6] E. Berkson and T.A. Gillespie, Stečkin's theorem, transference, and spectral decompositions, J. Functional Analysis 70 (1987), 140-170. MR 87m:47082

[7] E. Berkson and T.A. Gillespie, The spectral decomposition of weighted shifts and the $A_{p}$ condition, Colloquium Math. 60-61 (special issue dedicated to A. Zygmund) (1990), 507518. MR 92e: 47045

[8] E. Berkson and T.A. Gillespie, La q-variation des fonctions et l'intégration spectrale des multiplicateurs de Fourier, Comptes rendus de l'Académie des Sciences (Paris) t. 318, Série I (1994), 817-820. MR 95a:43005

[9] A.P. Calderón, Ergodic theory and translation-invariant operators, Proc. Nat. Acad. Sci. U.S.A. 59 (1968), 349-353. MR 37:2939

[10] R.R. Coifman and G. Weiss, Operators associated with representations of amenable groups, singular integrals induced by ergodic flows, the rotation method and multipliers, Studia Math. 47 (1973), 285-303. MR 49:1009

[11] J.L. Doob, Stochastic Processes, Wiley \& Sons, New York, 1953. MR 15:445b

[12] R.E. Edwards and G.I. Gaudry, Littlewood-Paley and Multiplier Theory, Ergebnisse der Math. und ihrer Grenzgebiete 90, Springer-Verlag, Berlin, 1977. MR 58:29760

[13] J. García-Cuerva and J.L. Rubio de Francia, Weighted Norm Inequalities and Related Topics, North-Holland Mathematics Studies $116=$ Notas de Matemática (104), Elsevier Science Publ., New York, 1985. MR 87d:42023

[14] T.A. Gillespie, A spectral theorem for $L^{p}$ translations, J. London Math. Soc. (2) 11 (1975), 499-508. MR 52:1398

[15] R. Hunt, B. Muckenhoupt, and R. Wheeden, Weighted norm inequalities for the conjugate function and Hilbert transform, Trans. Amer. Math. Soc. 176 (1973), 227-251. MR 47:701

[16] C.-H. Kan, Ergodic properties of Lamperti operators, Canadian J. Math. 30 (1978), 12061214. MR 80g: 47037

[17] D.S. Kurtz, Littlewood-Paley and multiplier theorems on weighted $L^{p}$ spaces, Trans. Amer. Math. Soc. 259 (1980), 235-254. MR 80f:42013

[18] J. Lamperti, On the isometries of certain function spaces, Pacific J. Math. 8 (1958), 459-466. MR 21:3764

[19] J. Lindenstrauss and L. Tzafriri, Classical Banach Spaces I (Sequence Spaces), Ergebnisse der Math. und ihrer Grenzgebiete 92, Springer-Verlag, Berlin, 1977. MR 58:17766

[20] M. Lorente Domínguez and F.J. Martín-Reyes, The ergodic Hilbert transform for Cesàro bounded flows, Tôhoku Math. J. 46 (1994), 541-556. MR 95k:28037 
[21] F.J. Martín-Reyes and A. de la Torre, The dominated ergodic theorem for invertible, positive operators, Semesterbericht Funktionalanalysis Tübingen, Sommersemester 1985, pp. 143-150.

[22] F.J. Martín-Reyes and A. de la Torre, The dominated ergodic estimate for mean bounded, invertible, positive operators, Proc. Amer. Math. Soc. 104 (1988), 69-75. MR 89i:47015

[23] B. Muckenhoupt, Weighted norm inequalities for the Hardy maximal function, Trans. Amer. Math. Soc. 165 (1972), 207-226. MR 45:2461

[24] R. Sato, A remark on the ergodic Hilbert transform, Math. J. Okayama Univ. 28 (1986), 159-163. MR 88k:47043

[25] E. Sawyer, Weighted inequalities for the one-sided Hardy-Littlewood maximal functions, Trans. Amer. Math. Soc. 297 (1986), 53-61. MR 87k:42018

Department of Mathematics, University of Illinois, 1409 West Green St., Urbana, ILLINOIS 61801

E-mail address: berkson@symcom.math.uiuc.edu

Department of Mathematics, University of Edinburgh, James Clerk Maxwell BuildING, Edinburgh EH9 3JZ, ScOtLAND

E-mail address: t.a.gillespie@edinburgh.ac.uk 